\title{
ESTUDO TEÓRICO, ATRAVÉS DA TEORIA DO FUNCIONAL DA \\ DENSIDADE, DAS PROPRIEDADES ÓPTICAS, ELETRÔNICAS E ESTRUTURAIS DO ALAS, DO TIPO FCC, VARIANDO A CONCENTRAÇÃO DA IMPUREZA TL
}

\author{
Carlos Rodolfo Bomfim Lopes Souza ${ }^{1}$; Nilton Souza Dantas ${ }^{2}$ \\ 1. Bolsista PROBIC/UEFS, Graduando em Bacharelado em Física, Universidade Estadual de Feira de Santana, e- \\ mail: crblsouza@gmail.com \\ 2. Orientador, Departamento de Exatas, Universidade Estadual de Feira de Santana, e-mail: nilton.dantas@gmail.com
}

PALAVRAS-CHAVE: DFT; propriedades eletrônicas; propriedades ópticas.

\section{INTRODUÇÃO}

Os novos materiais funcionais (assim chamados porque não se encontram na natureza, sendo obtidos por tecnologias de engenharia de materiais) constituem um importante ramo de pesquisa em ciência e engenharia de materiais por conta de sua aplicabilidade na indústria. Dentre esses materiais, os do grupo III-V da tabela periódica são os que têm mais se destacado, como por exemplo o Nitreto de Gálio (GaN). Esses materiais possuem uma característica especial que os tornam interessantes para aplicações em dispositivos optoeletrônicos: o gap.

O gap é uma das propriedades mais importantes nos semicondutores, porque determina a transição eletrônica entre os estados de energia, que é mediada principalmente pela radiação eletromagnética (Rezende, 2014). Assim, o controle do gap dos materiais semicondutores permite a criação de dispositivos como os lasers, células fotovoltaicas, detectores de radiação, entre outros. Um dos métodos utilizados para controlar o gap é atraveś da introdução de impurezas no material, num processo chamado de dopagem, em que há a inserção átomos de elementos diferentes daqueles que compõem a rede do material. De acordo com estudos teóricos recentemente publicados Dantas et al. (2008) e Silva et al. (2005), a inclusão de átomos de tálio ( $\mathrm{Tl}$ ) nesses materiais provoca a diminuição do gap. Estudos teóricos conduzidos anteriormente pelos autores também sugerem que a introdução de $\mathrm{Tl}$ em Arseneto de Alumínio (AlAs) provoca uma diminuição do gap, além de outras modificações nas propriedades eletrônicas e estruturais material. Dessa forma, isso o abre um novo campo de investigação no subconjunto dos materiais III-V, pois a introdução de uma impureza, tal como Tl, pode ser usada para controlar as propriedades desses materiais, tornando-os materiais funcionais. O controle do gap, por exemplo, pode permitir que sejam fabricados materiais cuja absorção de radiação possa variar, permitindo criar materiais com diversas aplicabilidades apenas controlando a inclusão da impureza Tl.

Foram feitas simulações em computador de um cristal de AlAs dopado com Tl usando a Teoria do Funcional da Densidade (DFT), descrita por Hohenberg \& Kohn (1964) e por Kohn \& Sham (1965), implementada no software WIEN2k por Blaha, Schwarz et al. (2013), sendo possível calcular as propriedades de interesse, como densidade de estados, gap óptico, a função dielétrica e as propriedades estruturais do material. 


\section{METODOLOGIA}

Foi usado o pacote simulação computacional de materiais WIEN2k, instalado no cluster de computadores (baseado na plataforma Rock Clusters) do Laboratório de Modelagem e Simulação Computacional de Novos Materiais no Departamento de Ciências Exatas. Foram modeladas células de arseneto de alumínio dopado com tálio $\left(\mathrm{Al}_{1-\mathrm{x}} \mathrm{Tl}_{\mathrm{x}} \mathrm{As}\right)$, onde $\mathrm{x}$ representa a concentração de $\mathrm{Tl}$ em \%, nas seguintes concentrações: $0 \%, 25 \%, 50 \%$, $75 \%$ e $100 \%$. Os cálculos das propriedades ópticas, eletrônicas e estruturais foram feitos usando a DFT através do método LAPW (Sing \& Nordström, 2006), utilizando a Aproximação do Gradiente Generalizado (PBE-GGA) (Perdew, Burke \& Ernzerhof, 1996), tal como implementado no software WIEN2k.

1 - Foi executado o cálculo da densidade de estados para cada concentração de $\mathrm{Tl} \mathrm{em}$ $\mathrm{Al}_{1-\mathrm{x}} \mathrm{Tl}_{\mathrm{x}} \mathrm{As}$. Essa etapa exigiu um bom controle na definição do número de pontos do espaço recíproco;

2 - Foi feito o cálculo da função dielétrica para cada concentração de $\mathrm{Tl}$ em $\mathrm{Al}_{1-\mathrm{x}} \mathrm{Tl}_{\mathrm{x}} \mathrm{As}$. Essa etapa exigiu um rigoroso controle na definição do número de pontos do espaço recíproco, posto que devem ser identificados os pontos críticos dessa função. Esses pontos críticos estão relacionados a transições eletrônicas específicas do material estudado. Essa etapa demandou muito trabalho computacional;

3 - Foi executado o cálculo das constantes elásticas para cada concentração de $\mathrm{Tl}$ em $\mathrm{Al}_{1-\mathrm{x}} \mathrm{Tl}_{\mathrm{x}} \mathrm{As}$. As constantes elásticas dos materiais foram obtidas fazendo-se pequenas deformações nos cristais, variando os parâmetros da rede. Foram calculadas as energias totais para vários tipos de deformações e através da lei de Hooke, obtém-se as relações entre as constantes elásticas, as energias totais e as deformações (Kittel, 2006). Com auxílio da equação de estado de Birch-Murnaghan (Murnaghan, 1944), foi feito um ajuste da curva Energia x Volume para as diversas deformações feitas.

A pesquisa bibliográfica também foi parte integrante desse trabalho e foi feita durante a duração do mesmo. Até o momento não são conhecidos resultados sobre o tema em estudo, porém foi necessário monitorar as publicações a fim de prever a tendência das pesquisas nesse campo, bem como comparar os resultados obtidos com outros análogos já publicados.

\section{RESULTADOS}

A tabela 1 mostra as constantes elásticas c11, c12 e c44, expressas em GPa, para cada célula cristalina.

Tabela 1. Constantes elásticas para cada concentração x de Tl.

\begin{tabular}{|c|c|c|c|}
\hline Concentração (x) & C11 (GPa) & C12 (GPa) & C44 (GPa) \\
\hline $0 \%$ & 113.2128 & 42.5606 & 28.7505 \\
\hline $25 \%$ & 89.5616 & 43.7676 & 33.5021 \\
\hline $50 \%$ & 72.6834 & 39.4190 & 24.5271 \\
\hline $75 \%$ & 65.3206 & 37.2815 & -21.4083 \\
\hline $100 \%$ & 44.1507 & 35.0376 & 30.5620 \\
\hline
\end{tabular}


Com as constantes elásticas, o módulo de compressibilidade $\mathrm{B}$ também foi calculado, variando, aproximadamente, linearmente com a concentração da impureza Tl. A figura 1 mostra a variação de $\mathrm{B}_{0}$ com a concentração de $\mathrm{Tl}$, o que sugere que TlAs é mais compressível que o AlAs.



Figura 1: variação do módulo de compressibilidade com a concentração de tálio

Foram calculadas as densidades de estados totais e parciais para cada material. Como já conhecido na literatura, o gap fundamental do material diminui com a introdução de Tl. Isso ocorre principalmente devido a contribuição das bandas 6 s e $6 \mathrm{p}$ do $\mathrm{Tl}$, que introduz novos estados na região próximo ao nível de Fermi.

Os cálculos da função dielétrica mostram que o pico de absorção do AlAs ocorre em 4,42 eV. Os gráficos da parte imaginária da função dielétrica mostram os picos de absorção. Os picos de energia na faixa entre 4 e $6 \mathrm{eV}$ correspondem as transições eletrônicas entre as bandas $4 \mathrm{~s}$ e $4 p$ do As e $3 \mathrm{~s}$ e $3 p$ do Al, como encontrado no cálculo da densidade de estados parcial. Como quase não há estados entre o topo da banda de valência e o fundo da banda de condução, há poucas transições na faixa de $2 \mathrm{eV}$.
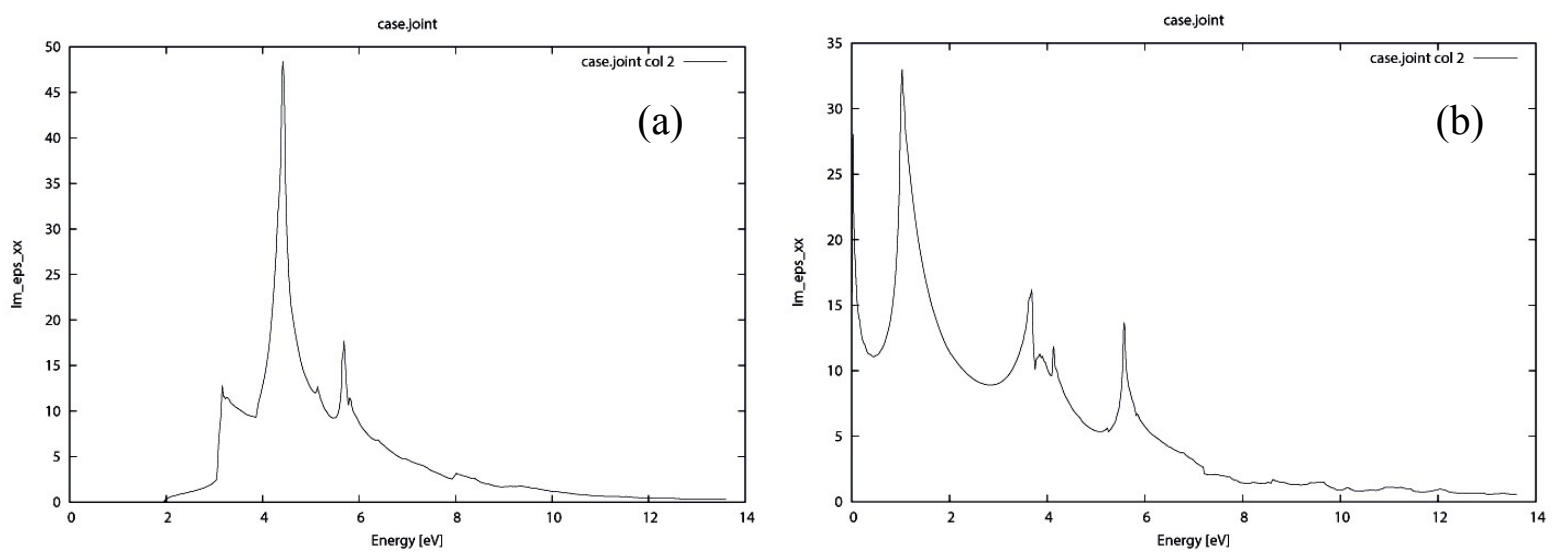

Figura 2: parte imaginária da função dielétrica para

(a) AlAs; (b) TlAs 
Com a introdução de Tl no material, há um deslocamento do pico para regiões de menores energias. Isso se deve porque o gap, que passa a ser direto, diminui, e há um aumento no número de estados na região próxima ao nível de Fermi. A análise do gráfico da parte imaginária função dielétrica do TlAs mostra que o pico de absorção ocorre principalmente em 2 pontos, de 0 a 1,5 eV, que corresponde a transições entre as bandas $6 \mathrm{~s}$ e $6 \mathrm{p}$ do $\mathrm{Tl}$ e $4 \mathrm{p}$ do As. Essas são as bandas que mais contribuem com o número de estados na região entre -2 e $2 \mathrm{eV}$.

\section{CONSIDERAÇÕES FINAIS}

Neste trabalho foi feito um estudo utilizando métodos em primeiros princípios sobre as propriedades eletrônicas, ópticas e estruturais do arseneto de alumínio dopado com tálio, usando a DFT, com funções de base LAPW, tal como implementada no software WIEN2k. Os cálculos das constantes elásticas e da estrutura de bandas foram feitos com o potencial de troca correlação PBE-GGA.

Os resultados encontrados mostram que a inclusão de tálio no arseneto de alumínio torna a célula cristalina maior e mais compressível. Isso já era esperado, pois foi verificado em um estudo anterior, não reportado aqui, que o parâmetro de rede cresce linearmente com a concentração de $\mathrm{Tl}$, obedecendo a regra de Vergard, e o material assume caráter mais metálico.

Os resultados sugerem que a diminuição do gap óptico com o aumento da concetração de tálio é causado por contribuições da bandas $6 \mathrm{~s}$ e $6 \mathrm{p}$ do tálio, que introduz novos estados na região de energia próximo ao nível de Fermi. Isso provoca um deslocamento do pico de absorção da função dielétrica para regiões de menor energia, de 4,42 eV no AlAs para 0,03 e 1,03 eV no TlAs. Assim, a introdução do tálio como dopante pode contribuir com o controle das transições eletrônicas desse material, podendo-se modificar o gap e consequentemente a condutividade e a resposta óptica linear.

Até aonde o momento não foram encontrados resultados na literatura sobre as propriedades estudadas desse material.

\section{REFERÊNCIAS}

REZENDE, S. M. 2014. Materiais e Dispositivos Eletrônicos, 3a ed., Editora Livraria da Física,São Paulo.

DANTAS, N. S. et al, 2008. App. Phys. Lett.,Vol. 92, 121914.

SILVA, A. F. da et al, 2005. Proc. of Int. Bulk Nitride Semicond. (Krakow, 2004), J. Cryst. Growth 281, 151.

HOHENBERG, P., KOHN, W., 1964. Phys. Rev. B 136864.

KOHN, W., SHAM, L. J. 1965. Phys. Rev. 140, A1133.

BLAHA, P., SCHWARZ, K., MADSEN, G. K. H., KVASNICKA, D., LUITZ, J. 2013. Wien2k, An Augmented Plane Wave + Local Orbitals Program for Calculating Crystal

Properties (Karlheinz Schwarz, Techn. Universität Wien, Austria), ISBN 3-9501031-1-2, revised edition.

SING D., NORDSTRÖM, L. 2006. Planewaves, pseudopotentials and LAPW method, $2^{\circ}$ ed. New york: Springer.

PERDEW, J. P., BURKE, K., ERNZERHOF, M. 1996. Phys. Rev, Lett., 773865.

KITTEL, C. 2006. Introdução à Física do Estado Sólido, 8a ed., LTC, São Paulo.

MURNAGHAN, F. D. 1944. Proc. Natl. Acad. Sci. USA 305188. 